\title{
Cooperative nucleation leading to ripple formation in InGaAs/GaAs films
}

\author{
Nehal S. Chokshi and Joanna Mirecki Millunchick ${ }^{\mathrm{a})}$ \\ Department of Materials Science and Engineering, University of Michigan, Ann Arbor, \\ Michigan 48109-2136
}

(Received 23 December 1999; accepted for publication 28 February 2000)

\begin{abstract}
$\mathrm{In}_{0.25} \mathrm{Ga}_{0.75} \mathrm{As}$ epilayers were grown on GaAs (001) substrates (1.8\% misfit strain) by molecular beam epitaxy to investigate the two-dimensional to three-dimensional transition as a function of thickness $(t \leqslant 30 \mathrm{MLs})$. Tapping-mode atomic force micrographs show the evolution of the morphology as a function of thickness. As the film is deposited, the nucleation of 3D islands followed by cooperative nucleation of pits is observed. As the thickness increases, both islands and pits continue to nucleate and grow until they coalesce, resulting in a fully formed ripple morphology running along the $[1 \overline{1} 0]$. The ripples also exhibit a secondary alignment roughly along the $\langle 310\rangle$ which is attributed to the nucleation of islands with $\{136\}$ faces. (C) 2000 American Institute of Physics. [S0003-6951(00)00917-7]
\end{abstract}

A detailed understanding of the two-dimensional to three-dimensional (3D) transition in strained heteroepitaxial growth will have tremendous impact on issues such as improved epilayer uniformity and increased quantum dot homogeneity. It is well known that $3 \mathrm{D}$ roughening will occur in lattice mismatched films above some critical thickness. In the most simple picture, 3D islands nucleate following misfit dislocation formation. However, the experiments of Eaglsham and Cerullo ${ }^{1}$ showed that coherent islands can also develop. In addition, Cullis et $a .^{2}$ showed that a strained film can form surface undulations that partially relieve strain. Subsequent experiments have applied both of these phenomena in exciting and novel ways. For example, the formation of coherent 3D islands has been utilized in quantum dot devices without the deleterious effects of postgrowth processing. ${ }^{3}$ Also, surface undulations have been shown to be correlated to lateral composition modulation, ${ }^{4}$ yet another avenue to spontaneous quantum dimensional structures.

Recently, theoretical models based on continuum elasticity ${ }^{5,6}$ have attempted to elucidate the mechanisms for governing the roughening process, but experimental verification is still underway and a unified understanding of the morphological evolution has yet to emerge. This letter presents a study of the morphological evolution of $\operatorname{In}_{0.25} \mathrm{Ga}_{0.75} \mathrm{As}$ alloy layers on (001) GaAs substrates (misfit strain $\approx 1.8 \%$ ) as a function of thickness in order to investigate the roughening process.

All films were grown in an EPI930 molecular beam epitaxy system with solid sources for the group III and V species. The substrate temperature was monitored using an optical pyrometer. The $\mathrm{GaAs}(001)$ substrates were prepared by heating them to $600{ }^{\circ} \mathrm{C}$ under an As overpressure until a spotty pattern was observed, followed by deposition of a 4000 - $\AA$-thick GaAs buffer layer at $580^{\circ} \mathrm{C}$. The $\mathrm{In}_{0.25} \mathrm{Ga}_{0.75}$ As films were then deposited to various thickness at a growth rate of $R=0.77 \mathrm{ML} / \mathrm{s}$ and a substrate temperature of $T=510^{\circ} \mathrm{C}$ (except where noted otherwise) under an As overpressure of $1.1 \times 10^{-5}$ Torr. The composition was deter-

${ }^{a)}$ Electronic mail: joannamm@umich.edu mined by reflection high-energy diffraction (RHEED) oscillations prior to growth of the samples. In situ measurements of the epitaxial growth was carried out by monitoring the intensity of the RHEED specular spot. Films were studied $e x$ situ using a Digital Instruments tapping-mode atomic force microscope (AFM) with etched silicon probes (nominal radius $\approx 5-10 \mathrm{~nm})$.

During the growth of the $\operatorname{In}_{0.25} \mathrm{Ga}_{0.75}$ As layers, the intensity of the RHEED specular spot was monitored. Figure 1 shows a plot of the RHEED specular spot intensity as a function of deposition time. Three regimes in the growth are observed. When the $\mathrm{In}_{0.25} \mathrm{Ga}_{0.75}$ As growth begins, the specular intensity oscillates. This is the layer-by-layer growth regime (regime I). Beyond a thickness of 5 MLs the oscillations decay and the pattern dims but remains streaky; this is the roughening regime (regime II). At a thickness of approximately $20 \mathrm{MLs}$, the specular spot intensity increases as the RHEED pattern evolves into a strongly spotty pattern indicating that $3 \mathrm{D}$ islands have developed on the surface (regime III). At a thickness $t \geqslant 30 \mathrm{MLs}$, chevrons appear on the

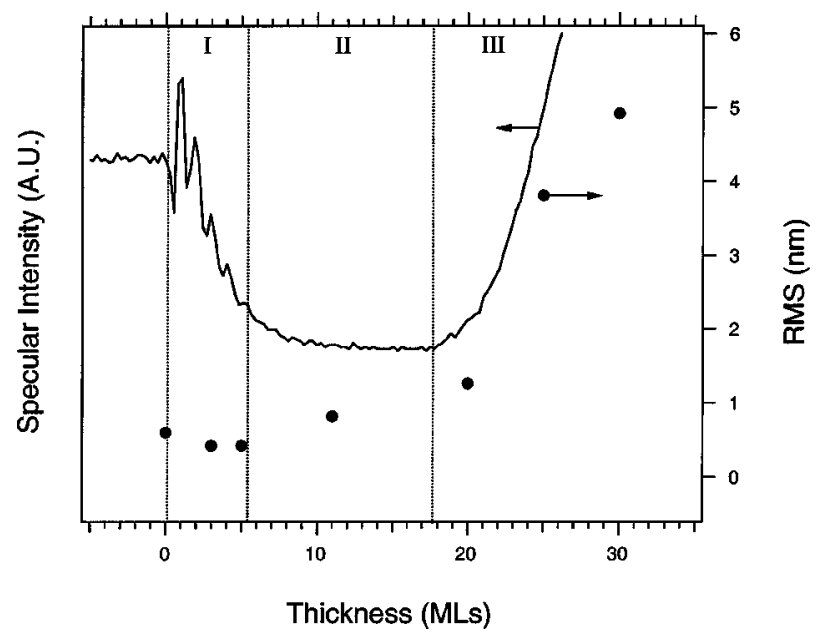

FIG. 1. RHEED specular spot intensity (line) and rms roughness (closed circles) as a function of deposited thickness. Both curves show that there are three regimes in the growth: (i) layer by layer, (ii) roughening, and (iii) 3D island nucleation. 
[1 $1 \overline{1} 0]$ azimuth. This evolution of the specular spot intensity is independent of the growth conditions. That is, changing parameters such as the growth temperature $(500<T$ $\left.<545^{\circ} \mathrm{C}\right)$ and the arsenic overpressure $\left(6 \times 10^{-6}<P_{\mathrm{As}_{4}}\right.$ $<1.2 \times 10^{-5}$ Torr) does not alter the fundamental regimes, however, the relative sizes of those regimes vary slightly.

The film morphology was characterized ex situ using AFM, and is consistent with the regimes observed by RHEED. The root-mean-square (rms) roughness for $4 \mu \mathrm{m}^{2}$ images is also shown in Fig. 1. The rms roughness is approximately constant in regime I, followed by a gradual increase in regime II. Once the growth crosses into regime III, the rms roughness increases very rapidly.

Figure 2 shows the morphological evolution of the films as a function of thickness. For $t=3 \mathrm{MLs}$ (not shown), the morphology consists of gently undulating mounds whose

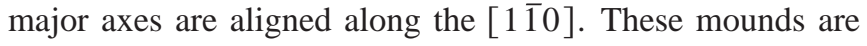
also observed in the buffer layer and are generally believed to be a result of unstable growth as a result of step edge barriers. ${ }^{7}$ Recently, it has been suggested that they are consequence of overgrowth of the rough surface resulting from thermal desorption of the native oxide layer. ${ }^{8}$ Adamczyk and co-workers have simulated homoepitaxial growth of GaAs on a surface with randomly distributed pits such as those formed during oxide desorption and found that mounds with dimensions similar to those observed here are formed. ${ }^{8}$

Figure 2(a) shows an image of an 11-ML-thick $\mathrm{In}_{0.25} \mathrm{Ga}_{0.75} \mathrm{As} / \mathrm{GaAs}$ film which falls in regime II of Fig. 1. The gentle undulations have evolved into flat-topped mesas. Linescans (not shown) clearly show that the height varies sinusoidally for the $t=3 \mathrm{ML}$ film, whereas they show that the height varies as a step function in the $t=11 \mathrm{ML}$ film. Also visible in the image is the presence of occasional islands (island density $N \approx 9 \times 10^{7} \mathrm{~cm}^{-2}$ ). Furthermore, the islands nucleate preferentially along the mesa edges. Despite the presence of widely scattered islands, this morphology is consistent with the streaky RHEED pattern observed when the growth was terminated.

Figure 2(b) shows the morphology of the film at $t$ $=20 \mathrm{ML}$, at the start of regime III. The RHEED pattern at the end of growth of this sample became spotty. The AFM micrograph shows a random distribution of quantum dots $\left(N \approx 9.9 \times 10^{9} \mathrm{~cm}^{-2}\right)$ consistent with previously reported observations. ${ }^{9}$ The islands are elongated along the [1 $\left.1 \overline{1} 0\right]$ direction with the ratio of the major to minor axes of the quantum dots $\approx 2: 1$. Also visible in this image are occasional pits that are decorated with quantum dots.

Figure 2(c) shows how this pit-island morphology continues to evolve. This AFM image is of a 51-ML-thick $\mathrm{In}_{0.25} \mathrm{Ga}_{0.75} \mathrm{As}$ films grown at $T=500$. This film is also in regime III, and the specular spot intensity behavior of this film is nominally equivalent to a 25 -ML-thick film grown at $T=510$. We present this data here even though it was not grown under the same growth conditions because the specular spot intensity behavior is consistent with the rest of the samples. In this image, several different features are distinctly observed: flat areas corresponding to the wetting layer, a high density of islands $\left(N \approx 1.87 \times 10^{10} \mathrm{~cm}^{-2}\right)$, and large pits. The islands possess a broad size distribution indicating that coalescence and coarsening has taken place. As in (a)

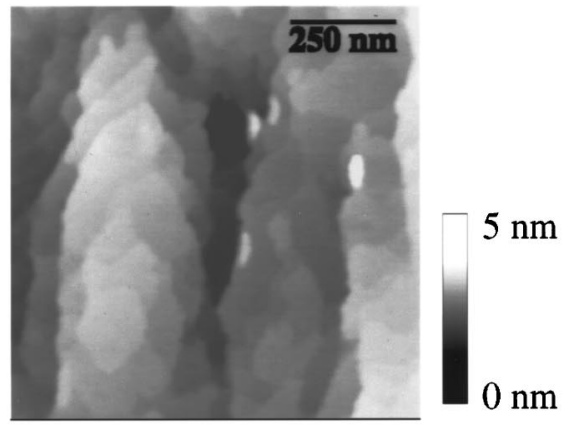

(b)

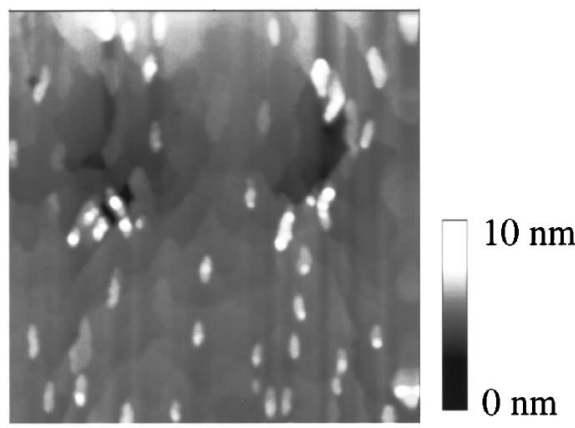

(c)
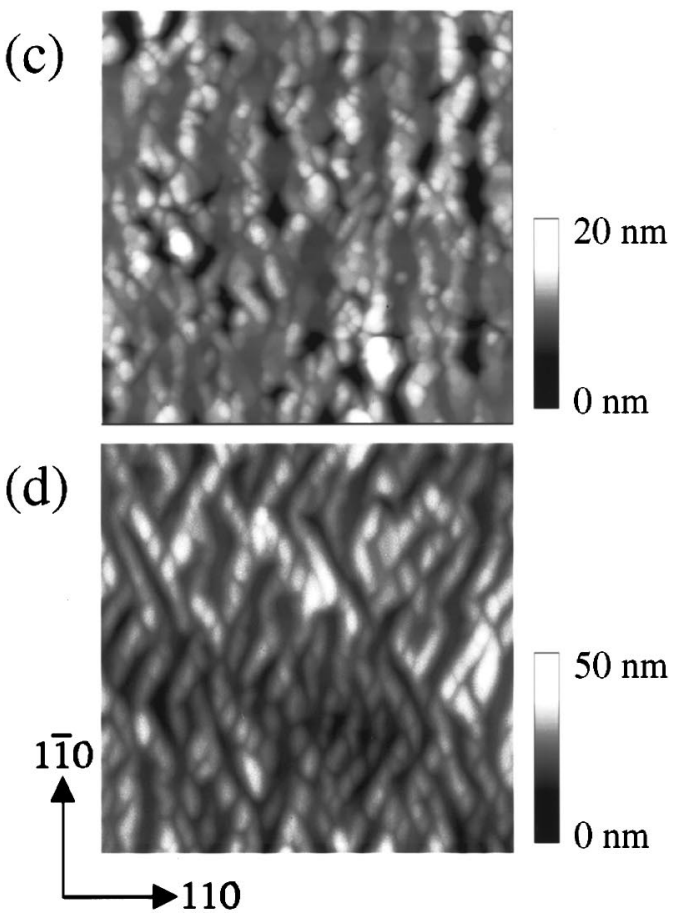

FIG. 2. Atomic force micrographs of the film morphology as a function of deposited thickness (a) Roughening and initial island nucleation in regime II ( $t=11 \mathrm{MLs}$ ), (b) quantum dot formation in the early states of regime III $(t=20 \mathrm{MLs})$, (c) cooperative nucleation in the intermediate stages of regime III, and (d) ripple formation in the later stages of regime III $(t$ $=30 \mathrm{MLs})$.

films of lower thickness, pits and islands appear to be clustered together, and they are aligned along the $[1 \overline{1} 0]$ direction.

Figure 2(d) shows an AFM image of a $t=30 \mathrm{ML}$ film which is well within regime III and exhibits a fully formed ripple morphology similar to that reported by Cullis and co-workers. ${ }^{2}$ The ripples are generally aligned parallel to the

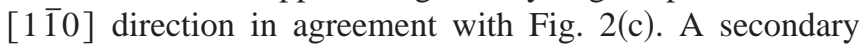
alignment is also observed that forms a diamond shaped pattern of features. Although the morphology consists of a network of ripples, discrete islands are still resolved. 
Whereas it has long been suggested that ripple arrays observed in systems with intermediate misfit strain ( $f$ $\approx 2 \%$ ) are formed from the coalescence of quantum dots, ${ }^{2}$ this work demonstrates that the cooperative nucleation of islands and pits is what leads to ripples. Figure 2(c) clearly shows three distinct features: islands, pits, and flat regions corresponding to the wetting layer. In the earliest stages of regime III where the RHEED specular intensity is increasing rapidly due to spot evolution, the rms roughness is also increasing rapidly. The data indicate that the roughening occurs not only by the nucleation and growth of islands, but also by the nucleation and growth of pits. Furthermore, the fact that islands and pits are adjacent to one another suggests that the islands grow by transferring material from the pits to the island.

This cooperative nucleation mechanism has also been observed in SiGe/Si alloys having similar misfit strain. ${ }^{10}$ Jesson and coworkers calculated that sequential nucleation of pits and islands is energetically more favorable over simultaneous formation of an extended perturbation, provided that the wetting layer is thick enough. Our data support this view. We observe that only islands nucleate at $t=11 \mathrm{MLs}$. However, we also observe that they preferentially form at mesa edges; i.e., next to trenches [Fig. 2(a)]. Island-trench pairs evolve into depressions ringed with islands at $t=20 \mathrm{MLs}$, as seen in Fig. 2(b), before finally forming the extensive network of island-pit pairs as seen in Fig. 2(c). This implies that the wetting layer for the $t<20 \mathrm{ML}$ films is insufficient to support pit nucleation. Once that thickness is reached, however, pit nucleation and growth can be sustained and the ripple array formation progresses rapidly.

Another interesting feature of these ripple arrays is the direction along which they form. It has been long suggested that the anisotropic alignment of ripple arrays in III-V systems has been due to the presence of a fast diffusion direction in the $[1 \overline{1} 0]$ due to the surface reconstruction. ${ }^{11} \mathrm{We}$ propose that the anisotropy is not due to the reconstruction alone. Rather, the observed asymmetry may also be due to the presence of facets on the islands and pits. Fig. 2(d) clearly shows that the ripples form a diamond shaped pattern. Fast Fourier transforms of the image show that the angle between the two secondary alignment directions is $53^{\circ}$. This is compatible with alignment along the [130] and [310] directions, which is consistent with an island that is bounded by $\{136\}$ faces. ${ }^{12,13}$ This secondary alignment is also apparent although not properly identified in previously published work. ${ }^{2}$ Such an island has a diamond shape elongated along the $[1 \overline{1} 0]$ with base edges parallel to the $\langle 310\rangle$. Therefore, a close packed arrangement of islands having this shape will also have a primary alignment in the [1 $1 \overline{0}]$ with a secondary alignment in the $\langle 310\rangle$. Note also that such an island shape is predicted to have an elongated shape with the diameter in the [11̄0] twice that of the diameter in the [110] $\left(d_{1 \overline{1} 0}\right.$ $\left.=2 d_{110}\right)$. Careful analysis of the quantum dots in Fig. 2(b) shows that $\left\langle d_{110}\right\rangle=95 \mathrm{~nm}$ and $\left\langle d_{110}\right\rangle=52 \mathrm{~nm}$, in good agreement with this proposed island shape. A statistical analysis of the feature sizes shows that the feature size (either island or pit) is nominally pinned in the [110] direction, but not in the $[1 \overline{1} 0]$ which is also consistent with the tiling of such diamond shaped islands. The explanation for why these faces posses the lowest energy is not known and is under investigation.

In summary, we have shown that the morphological evolution of $\operatorname{In}_{0.25} \mathrm{Ga}_{0.75} \mathrm{As}$ films has three general regimes that are independent of the growth conditions. They are characterized by layer-by-layer growth, roughening, and 3D growth. Within the roughening regime, widely separated quantum dots nucleate first at step edges, followed by the cooperative nucleation of both islands and pits in regime III. The islands and pits eventually coalesce into ripple arrays that are primarily aligned along the [1 10$]$ direction, with a secondary alignment along the $\langle 130\rangle$.

The authors wish to thank Professor B. G. Orr and Professor L. M. Sander for helpful discussions. This research is sponsored by the Division of Materials Research, National Science Foundation under Contract No. DMR-9973352.

${ }^{1}$ D. J. Eaglesham and M. Cerullo, Phys. Rev. Lett. 64, 1943 (1990).

${ }^{2}$ A. G. Cullis, A. J. Pidduck, and M. T. Emeny, J. Cryst. Growth 158, 15 (1996).

${ }^{3}$ A. E. Zhukov, V. M. Ustinov, A. Y. Egorov, A. R. Kovsh, A. F. Tsatsul'nikov, M. V. Maximov, N. N. Ledentsov, S. V. Zaitsev, N. Y. Gordeev, V. I. Kopchatov, Y. M. Shernyakov, P. S. Kop'ev, D. Bimberg, and Z. I. Alferov, J. Electron. Mater. 27, 106 (1998).

${ }^{4}$ J. Mirecki-Millunchick, R. D. Twesten, S. R. Lee, D. M. Follstaedt, E. D. Jones, S. P. Ahrenkiel, Y. Zhang, H. M. Cheong, and A. Mascarenhas, J. Electron. Mater. 26, 1049 (1997).

${ }^{5}$ J. E. Guyer and P. W. Voorhees, J. Cryst. Growth 187, 150 (1998).

${ }^{6}$ F. Leonard and R. C. Desai, Phys. Rev. B 57, 4805 (1998).

${ }^{7}$ C. Orme, M. D. Johnson, K. T. Leung, and B. G. Orr, Mater. Sci. Eng., B 30, 143 (1995).

${ }^{8}$ M. Adamczyk, A. Ballstad, T. Pinnington, T. Tiedje, M. Davies, and Y. Feng, J. Vac. Sci. Technol. (in press).

${ }^{9}$ C. W. Snyder, B. G. Orr, D. Kessler, and L. M. Sander, Phys. Rev. Lett. 66, 3032 (1991).

${ }^{10}$ D. E. Jesson, K. M. Chen, S. J. Pennycook, T. Thundat, and R. J. Warmack, Phys. Rev. Lett. 77, 1330 (1996).

${ }^{11}$ K. Shiraishi, Appl. Phys. Lett. 60, 1363 (1992).

${ }^{12}$ H. Lee, R. Lowe-Webb, W. Yang, and P. C. Sercel, Appl. Phys. Lett. 72, 812 (1998).

${ }^{13}$ H. Lee, W. Yang, P. C. Sercel, and A. G. Norman, J. Electron. Mater. 28, 481 (1999). 